\title{
Organic magnetoresistance near saturation: mesoscopic effects in small devices
}

\author{
R. C. Roundy, Z. V. Vardeny, and M. E. Raikh \\ Department of Physics and Astronomy, University of Utah, Salt Lake City, UT 84112
}

\begin{abstract}
In organic light emitting diodes with small area the current may be dominated by a finite number, $N$ of sites in which the electron-hole recombination occurs. As a result, averaging over the hyperfine magnetic fields, $\boldsymbol{b}_{h}$, that are generated in these sites by the environment nuclei is incomplete. This creates a random (mesoscopic) current component, $\delta I(\boldsymbol{B})$, at field $\boldsymbol{B}$ having relative magnitude $\sim N^{-1 / 2}$. To quantify the statistical properties of $\delta I(\boldsymbol{B})$ we calculate the correlator $K\left(\boldsymbol{B},{ }_{\Delta} \boldsymbol{B}\right)=\left\langle\delta I\left(\boldsymbol{B}-\frac{\Delta \boldsymbol{B}}{2}\right) \delta I\left(\boldsymbol{B}+\frac{\Delta \boldsymbol{B}}{2}\right)\right\rangle$ for parallel, $\Delta \boldsymbol{B} \| \boldsymbol{B}$, and perpendicular, $\Delta \boldsymbol{B} \perp \boldsymbol{B}$ orientations of $\Delta \boldsymbol{B}$. We demonstrate that mesoscopic fluctuations develop at fields $|\boldsymbol{B}| \gg\left|\boldsymbol{b}_{h}\right|$, where the average magnetoresistance is near saturation. These fluctuations originate from the slow beating between the singlet, $S$ and triplet, $T_{0}$ states of the recombining $e-h$ spin pair-partners. We identify the most relevant processes responsible for the current fluctuations as due to anomalously slow beatings that develop in sparse $e-h$ polaron pairs at sites for which the $\boldsymbol{b}_{h}$ projections on the external field direction almost coincide.
\end{abstract}

PACS numbers: 73.50.-h, 75.47.-m

\section{INTRODUCTION}

In the field of 'Dynamic Spin Chemistry', a mechanism by which the recombination rate of radical pairs is sensitive to a weak magnetic field, $\boldsymbol{B}$, was established more than four decades ago; see, e.g., the reviews in Ref. 1 . This mechanism relies on the hyperfine interaction of the spin-1/2 pair partners with their respective nuclear spin environments, where the hyperfine field, $\boldsymbol{b}_{h}$ generated by the nuclei is responsible for the radical spins dynamics in zero field. In this process if at time $t=0$ the radical pair spin state is, e.g., in a singlet configuration, $S$, then at finite $t$ it will acquire a triplet $(T)$ component with probability, $P_{S T}(t)$. If recombination is allowed only from $S$, then $P_{S T}(t)$ dynamic evolution affects the recombination rate. Clearly, $P_{S T}(t)$ depends on $\boldsymbol{B}$ and this sets a small scale, $|\boldsymbol{B}| \sim\left|\boldsymbol{b}_{h}\right|$ that may influence the radical pair recombination rate.

An important advance in the quantitative description

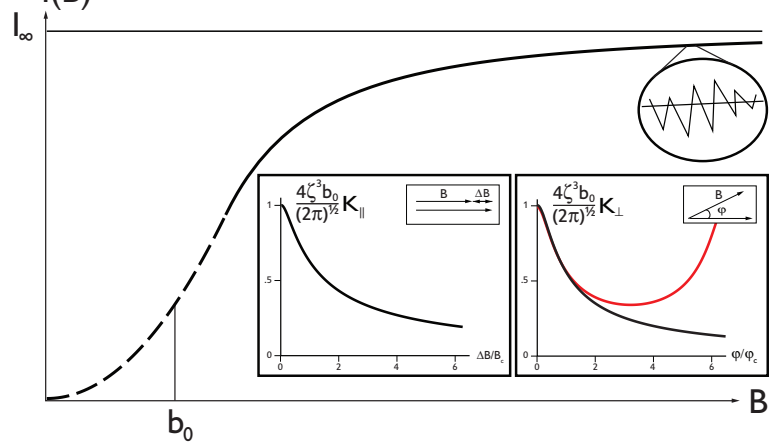

FIG. 1: The dependence $I(B)$ of the device current on the applied magnetic field is shown schematically in the strong-field limit $B \gg b_{0}$. Enlargement illustrates mesoscopic fluctuations emerging in a small sample. Two insets are the correlators of the mesoscopic fluctuations for $\Delta \boldsymbol{B} \| \boldsymbol{B}$ and $\Delta \boldsymbol{B} \perp \boldsymbol{B}$ plotted from Eqs. (13) and (15), respectively .

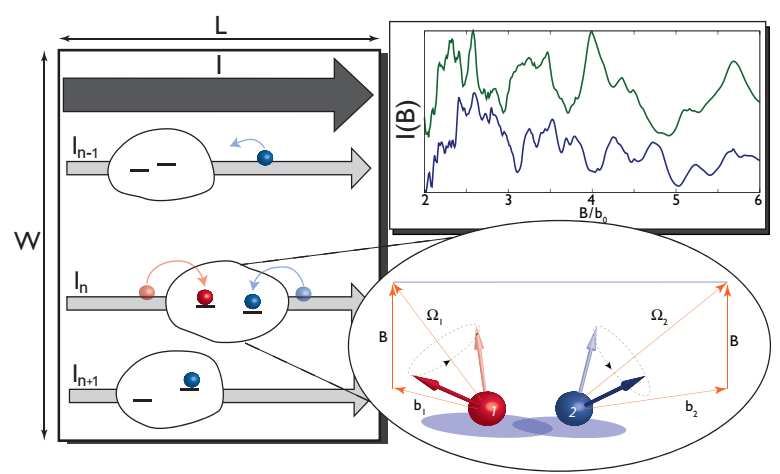

FIG. 2: In strongly inhomogeneous device with $W \gg L$ the current passage is dominated by the most conductive channels, $I=\sum_{n} I_{n}$. Each current component is limited by the most resistive junction, illustrated schematically. The current through this junction is sensitive to the spin dynamics of the constituting PP. "Slow" pairs, shown in the enlargement, are those in which the $z$-projections of their hyperfine fields coincide accidentally. The inset shows $\sum_{n=1}^{N} I_{n}$ calculated for two realizations of $N=10^{4}$ random hyperfine fields with $\mathrm{rms}$ $b_{0}=10^{2} \zeta^{-1}$.

of $P_{S T}(t)$ was made by Schulten and Wolynes 2 . They noticed that, due to the large number of nuclei surrounding each radical pair, and slow dynamics of the hyperfine field, the $\boldsymbol{b}_{h}$ random distribution may be modeled by a Gaussian. Under these conditions the multiplicity of the nuclear spin configurations may be characterized by a single number - namely the width of this distribution, $b_{0}$.

The dependence of $P_{S T}(t)$ on $\boldsymbol{B}$ is at the core of organic magnetoresistance (OMAR), which has recently attracted a lot of attention ${ }^{3-18}$. This is because the current, $I$ in a biased organic diode involves recombination of the injected $e-h$ polarons (forming polaron pairs, PP); whereas the processes of populating and depopulating of traps are not sensitive to spin dynamics.

The theory of OMAR is conceptually harder than that 
of spin-magneto-chemistry ${ }^{\underline{1}}$ for two reasons. Firstly, in OMAR the complex dynamics of all four PP spin states, $S, T_{0}, T_{+}$, and $T_{-}$needs be incorporated into the calculation of the dc current that is influenced by the PP. Secondly, each PP is sensitive to the other PPs if they belong to the same current path. Finally, averaging over the nuclear environment should be carried out only at the last step. To bypass these complications several simplifying assumptions concerning both the spin dynamics and current passage scenario were adopted in previous theoretical calculations of the $I(B)$ response $e^{6,12-18}$.

In contrast, in the present paper we do not focus on the entire $I(B)$ response, but rather on the strong $B$ domain, where the OMAR response is close to saturation, see Fig. 1. Our motivation is twofold. Firstly, theory allows a dramatic simplification in this $B$-domain, since the spin dynamics that is relevant to OMAR involves only the PP $S$ and $T_{0}$ states. However even in this $B$-domain the OMAR underlying physics is not trivial if the hyperfine field is sufficiently strong; namely when $b_{0} \tau \gg 1$, which corresponds to the regime of "slow" hopping 16,18. Here $\tau$ is a characteristic recombination time, and $b_{0}$ is measured in frequency units. Experimentally 13 , in organic semiconductors $b_{0}$ is $\sim 1 \mathrm{mT}$, whereas $\tau \sim 1-10 \mu \mathrm{s}$, so that this parameter is $\sim 10^{3}$. We show that at large $b_{0} \tau$ the spin dynamics is not "frozen" as $B$ exceeds $b_{0}$, but persists in a parametrically broad interval, $b_{0}^{2} \tau \gg B \gg b_{0}$. Our second and central motivation for considering strong fields is that we predict the occurrence of mesoscopic properties in this $B$-domain that would form in small devices that are based on strongly disordered organic active layers. Specifically we predict reproducible random fluctuations in the $I(B)$ response upon sweeping $B$ (see Fig. 1), which reflect the "individuality" ${ }^{19-21}$ of the nuclear environments associated with the relevant recombination centers in the organic.

More quantitatively, if the number, $N$ of current paths that contribute to $I(B)$ is finite, then the statistical averaging over $\boldsymbol{b}_{h}$ is incomplete. The relative fluctuation $\frac{\delta I(B)}{\langle I\rangle} \sim N^{-1 / 2}$ while small, can be still experimentally obtained because of the high accuracy with which current can be measured. In the field of 'dynamic spin chemistry', mesoscopic fluctuations cannot occur since the number of radical pairs that contribute to the observable characteristics is huge.

Obviously, the necessary condition to observe mesoscopic fluctuations in the $I(B)$ response of organic devices is slow nuclear spin dynamics, which should allow one to obtain $I(B)$ before the nuclear configuration changes. This is realistic, since the characteristic time for current passage is a $\mathrm{PP}$ recombination time, which for organic devices is $50 \mu \mathrm{s}$, see Ref. 22. It is generally accepted ${ }^{2,23}$ that the time for the change of the nuclear-spin configuration is orders of magnitude longer, although no accurate measurements of proton spin-spin relaxation time for organic devices have yet been reported in the literature.

\section{PP DYNAMICS IN STRONG FIELDS}

\section{A. Isolated PP}

We start with a detailed account of the PP spin dynamics and recombination in the strong $B$-domain, which we then use to calculate mesoscopic contribution to $I(B)$ near saturation. For an isolated PP the spin Hamiltonian $\widehat{H}=\boldsymbol{\Omega}_{1} \cdot \widehat{\boldsymbol{S}}_{1}+\boldsymbol{\Omega}_{2} \cdot \widehat{\boldsymbol{S}}_{2}$ describes the precession of the PP spins $\boldsymbol{S}_{1}, \boldsymbol{S}_{2}$ in the fields $\boldsymbol{\Omega}_{1}=\boldsymbol{B}+\boldsymbol{b}_{1}$ and $\boldsymbol{\Omega}_{2}=\boldsymbol{B}+\boldsymbol{b}_{2}$, respectively. If at $t=0$ the $\mathrm{PP}$ is in the singlet state, then the probability, $P_{S S}(t)$ to find it in the singlet state at finite $t$ oscillates with time. $P_{S S}(t)$ oscillations contain two frequencies: $\Delta=\left|\boldsymbol{\Omega}_{1}\right|-\left|\boldsymbol{\Omega}_{2}\right|$ and $\Sigma=\left|\boldsymbol{\Omega}_{1}\right|+\left|\boldsymbol{\Omega}_{2}\right|$. The advantage in considering the strong-field limit is that since $\left|\boldsymbol{\Omega}_{1}\right| \approx\left|\boldsymbol{\Omega}_{2}\right|$, the frequencies $\Delta$ and $\Sigma$ are very different from each other, so that the spin dynamics decouples into distinct 'slow' and 'fast' modes. Moreover, the slow mode involves predominantly $S$ and $T_{0}$ states, while the admixture of $T_{+}$and $T_{-}$states to this mode is relatively weak (of the order of $b_{0}^{2} / B^{2}$ ). The fast mode $\Sigma$ has frequency $\approx 2 B$ and describes the oscillations between $S$ and $T_{+}, T_{-}$. But the admixture of $S$ to this mode is also suppressed as $b_{0}^{2} / B^{2}$ in the strong-field limit. We thus conclude that, with accuracy $b_{0}^{2} / B^{2}, P_{S S}(t)$ dynamics simplifies in the strong-field limit to $P_{S S}(t)=\cos ^{2} \Delta t$; namely the 'beating' between $S$ and $T_{0}$ states. Similarly, if in the strong-field limit the $\mathrm{PP}$ is initially in the $T_{0}$ state then the probability to find it in the $S$ state at time $t$ is $\sin ^{2} \Delta t$.

\section{B. Recombination in the presence of $S-T_{0}$ beating}

We now assume that the PP is still isolated from the 'leads', but can recombine from $S$ to the ground state, $G$. A crucial question for OMAR is: what are the waiting times $\langle t\rangle_{S},\langle t\rangle_{T_{0}}$ for the recombination, if the system is initially in $S$ and $T_{0}$, respectively. The simplified spin dynamics in the strong-field limit allows us to address this question analytically.

Upon restricting the basis to $S, T_{0}$ and the ground state, we have 9 relevant elements of the density matrix for solving the Liouville-Lindblad equations of motion: $\dot{\rho}=-i[\hat{H}, \rho]+\hat{L}(\rho)$, where the operator $\hat{L}(\rho)$ describes the recombination. To find, e.g. $\langle t\rangle_{S}$ the system should be solved with the initial conditions $\rho(0)=|S\rangle\langle S|$. Subsequently $\langle t\rangle_{S}$ is found from the formula

$$
\langle t\rangle_{S}=\int_{0}^{\infty} d t t \frac{\partial \rho_{G G}}{\partial t}=\int_{0}^{\infty} d t\left(\rho_{S S}(t)+\rho_{T_{0} T_{0}}(t)\right) .
$$

Similarly $\langle t\rangle_{T_{0}}$ is obtained from Eq. (1) upon solving the equations of motion with initial conditions $\rho(0)=$ $\left|T_{0}\right\rangle\left\langle T_{0}\right|$. These calculations yield

$$
\langle t\rangle_{S}=\tau, \quad\langle t\rangle_{T_{0}}=\tau+\frac{1}{2 \tau \Delta^{2}}
$$




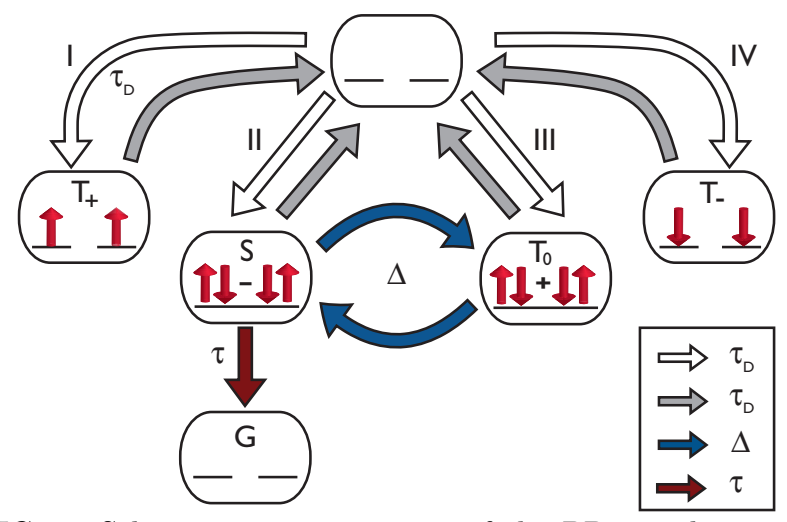

FIG. 3: Schematic representation of the PP population dynamics in the strong-field domain. For variants of cycle I (IV) the pair is assembled and, subsequently, disassembled in $T_{+}$ $\left(T_{-}\right)$state. For variants II (III) the pair is assembled in $S$ $\left(T_{0}\right)$ state in which it undergoes slow dynamics prior to disassembly. In the course of the slow dynamics the pair can recombine; recombination is possible only from $S$. Since the transport is unidirectional, current is passed through a junction upon completion of each cycle variant.

For a typical PP we have $\Delta=\left|\boldsymbol{\Omega}_{1}\right|-\left|\boldsymbol{\Omega}_{2}\right| \sim b_{0}$. Eq. (2) suggests that $\langle t\rangle_{S} \approx\langle t\rangle_{T_{0}} \approx \tau$. This is a natural result since recombination is preceded by many beatings between $S$ and $T_{0}$ states; therefore the recombination time does not depend on the initial PP state. The most striking consequence of Eq. (2) is that for sparse PP for which $\Delta$ is accidentally smaller than $\tau^{-1}$ we have $\langle t\rangle_{S} \ll\langle t\rangle_{T_{0}} \approx \frac{1}{2 \Delta^{2} \tau}$. This suggests that the smaller is $\Delta$, the longer the pair stays "trapped" in $T_{0}$. Note that in the course of beating without possibility of recombination, such PP would cross from $T_{0}$ to $S$ after much shorter time $\Delta^{-1} \ll\langle t\rangle_{T_{0}}$. We can trace the origin of the "trapping" described by Eq. (2) to the complex eigenmodes of the system that consists of singlet and triplet components being mixed by the hyperfine field. This system may be described by the $2 \times 2$ non-hermitian matrix:

$$
\begin{aligned}
& |S\rangle \quad|T\rangle \\
& \left(\begin{array}{cc}
-i / \tau & \Delta \\
\Delta & 0
\end{array}\right),
\end{aligned}
$$

where the nondiagonal elements describe the mixing, while $-i / \tau$ describes recombination from $S$ to $G$. The eigenvalues of this matrix are

$$
\lambda_{1,2}=-\frac{i}{2 \tau} \pm \sqrt{\Delta^{2}-\frac{1}{4 \tau^{2}}} .
$$

In the limit $\Delta \ll \tau^{-1}$ we have $\lambda_{1} \approx-\frac{i}{\tau}$, while $\lambda_{2} \approx$ $i \tau \Delta^{2}$. We see that $\lambda_{2}$ is anomalously small, and the result Eq. (22) for $\Delta \ll \tau^{-1}$ can be interpreted as $\langle t\rangle_{T_{0}} \sim \frac{1}{\lambda_{2}}$. We note in passing that the emergence of slow mode, $\lambda_{2}$ in a compound system with anomalously close levels was previously found in Refs. 2426 in connection with resonant tunneling through pairs of localized states.

Eqs. (3) and (4) provide a semi-qualitative derivation of our main result Eq. (2). A detailed derivation which justifies the above procedure is presented in Ref. 27 for the case of arbitrary external field. In particular, this paper deals with a delicate fact that the eigenvectors of non-hermitian matrix Eq. (3) are not orthogonal to each other.

In the remainder of the paper we demonstrate that it is the sparse pairs with $\Delta \lesssim \tau^{-1}$ that are responsible for the mesoscopic part of the $I(B)$ response in the strongfield limit.

\section{TRANSPORT MODEL}

We assume that the organic active layer in the device is strongly inhomogeneous and its width, $W$ is much larger than the distance, $L$ between the electrodes, see Fig. 2. Under these conditions the minimal description of transport is to model the sample as $N \gg 1$ parallel conducting channels. Moreover due to the film inhomogeneity, the current through each channel is limited by a single, most resistive junction. The stronger is the inhomogeneity, the more realistic is the proposed model, see e.g. the review Ref. 20. The net current through the sample is the sum, $I=\sum_{n=1}^{N} I_{n}$, of the currents in each channel. Each junction, $n$, can be viewed as a pair of sites coupled to the nuclei environment. In the course of the current $I_{n}$ through the junction, the pair of sites first gets occupied, is then emptied, and so on. In other words, the current passage can be viewed as a sequence of cycles, see Fig. 3. Each cycle consists of two steps, namely assembly of a pair on neighboring sites and disappearance of the pair through either dissociation or recombination, see Fig. 3. At this point we emphasize that it is the recombination stages of the cycles II and III (defined in Fig. 3) that are described by Eq. (2), and are thus sensitive to $B$.

The simplified transport model described in Fig. 2 encodes the same picture of transport put forward in Ref. 13. It contains recombination and bypassing, if recombination takes too long. From Fig. 3 we may write the average duration $\left\langle t_{n}\right\rangle$ of the cycle as follows

$$
\left\langle t_{n}\right\rangle=2 \times \frac{1}{4}\left(2 \tau_{D}\right)+\frac{1}{4}\left(\langle t\rangle_{S}+\tau_{D}\right)+\frac{1}{4}\left(\langle t\rangle_{T_{0}}+\tau_{D}\right) .
$$

The first term in Eq. (5) originates from the variants I and IV of the current cycle when the pair is assembled, and subsequently disassembled in $T_{+}$and $T_{-}$states, respectively. Then the cycle lasts for time $2 \tau_{D}$. The last two terms in Eq. (5) describe the current cycle variants II and III, in which the pair is assembled in either $S$ or $T_{0}$. Eq. (5) takes into account that realization of each current cycle has equal probability of $\frac{1}{4}$. For simplicity we assume that processes involving leads, namely, assembly and dissociation, take equal time, $\tau_{D}$. The current through the junction, which is the inverse cycle duration, can be then cast in the form

$$
I_{n}=\frac{1}{\left\langle t_{n}\right\rangle}=8 \zeta^{2} \tau-\delta I_{n}(\boldsymbol{B}), \quad \delta I_{n}(\boldsymbol{B})=\frac{8 \zeta^{4} \tau}{\Delta_{n}(\boldsymbol{B})^{2}+\zeta^{2}},
$$


where we used Eq. (2) and introduced the characteristic frequency

$$
\zeta=\frac{1}{2\left[\tau\left(3 \tau_{D}+\tau\right)\right]^{1 / 2}}
$$

We emphasize that the correction, $\delta I_{n}(\boldsymbol{B})$, in Eq. (6) originates from $S-T_{0}$ beating. For a typical nuclear environment we have $\Delta_{n} \sim b_{0}$, so that the relative magnitude of this correction is $\sim \frac{\zeta^{2}}{b_{0}^{2}} \ll 1$. However, on average, this term is much bigger, since it is dominated by sparse configurations with anomalously small $\Delta_{n} \sim \zeta$. This is because, while the portion of these sparse configurations is small, $\sim \zeta / b_{0}$, the $\delta I_{n}$ value for these configurations exceeds the typical $\delta I_{n}$ by a large factor $\frac{b_{0}}{\zeta}$. It can be demonstrated through a careful analysis 27 that the correction $\delta I_{n}(\boldsymbol{B})$ is insensitive to $\boldsymbol{B}$ on average. The number of "slow" pairs decreases with $B$. It was established in Ref. 27 that the PP recombination time also decreases with $B$ in such a way that the two tendencies compensate each other identically. In spite of this, it is the correction Eq. (66) that gives rise to the mesoscopic fluctuations of current to which we now turn.

\section{MESOSCOPIC FLUCTUATIONS}

If a given pair contributes to the correction $\delta I_{n}(\boldsymbol{B})$ in Eq. (6), then the $S-T_{0}$ splitting, $\Delta_{n}(\boldsymbol{B})$ for this pair is $\sim \zeta$. This suggests that, upon changing $\boldsymbol{B}$ by a small $\Delta \boldsymbol{B}$, the condition $\Delta_{n}(\boldsymbol{B}) \sim \zeta$ for this pair is violated, while it becomes satisfied for different pairs. Such "switching" of pairs contributing to the correction, $\delta I(\boldsymbol{B})$ gives rise to the mesoscopic fluctuations of the current, which we may quantify by the correlator

$$
K\left(\boldsymbol{B},{ }_{\Delta} \boldsymbol{B}\right)=\left\langle\delta I\left(\boldsymbol{B}-\frac{\Delta \boldsymbol{B}}{2}\right) \delta I\left(\boldsymbol{B}+\frac{\Delta \boldsymbol{B}}{2}\right)\right\rangle-\langle\delta I(\boldsymbol{B})\rangle^{2} .
$$

We consider two cases. In the first case, $\Delta \boldsymbol{B} \| \boldsymbol{B}$, the two magnetic fields are collinear. In the second case, $\Delta \boldsymbol{B} \perp$ $\boldsymbol{B}$, the two magnetic fields have the same magnitude but are rotated through an angle $\varphi$ with respect to each other.

For calculating the correlator in Eq. (8) we recast the factor $\left(\Delta^{2}+\zeta^{2}\right)^{-1}$ in $\delta I_{n}$ as a Fourier transform

$$
\frac{1}{\Delta^{2}+\zeta^{2}}=\frac{1}{2 \zeta^{2}} \int_{-\infty}^{\infty} d s \exp \left(-|s|+i \frac{s \Delta}{\zeta}\right) .
$$

By virtue of this transformation, the beating frequency $\Delta$, which depends on the hyperfine fields, appears in the exponent of the integrand. Next we take advantage of the fact that the beating frequency, $\Delta$ in the strong-field limit can be expanded as

$$
\Delta=\left(\boldsymbol{b}_{1}-\boldsymbol{b}_{2}\right) \cdot \boldsymbol{n}+\frac{\boldsymbol{b}_{1}^{2}-\boldsymbol{b}_{2}^{2}-\left(\boldsymbol{b}_{1} \cdot \boldsymbol{n}\right)^{2}+\left(\boldsymbol{b}_{2} \cdot \boldsymbol{n}\right)^{2}}{2 B},
$$

where $\boldsymbol{n}$ is the unit vector in the direction of $\boldsymbol{B}$. Now, since $\Delta$ contains only linear and quadratic terms in $\boldsymbol{b}_{i}$, the averaging of the exponential factor can be performed explicitly using the properties

$$
\left\langle e^{i \kappa b_{i}}\right\rangle=e^{-\kappa^{2} b_{0}^{2} / 4}, \quad\left\langle e^{-\mu b_{i}^{2}}\right\rangle=\frac{1}{\sqrt{1+\mu b_{0}^{2}}} .
$$

For the parallel case, this averaging yields

$$
\left\langle\frac{1}{\left(\Delta\left(\boldsymbol{B}+\frac{\Delta \boldsymbol{B}}{2}\right)^{2}+\zeta^{2}\right)\left(\Delta\left(\boldsymbol{B}-\frac{\Delta \boldsymbol{B}}{2}\right)^{2}+\zeta^{2}\right)}\right\rangle=\frac{1}{4 \zeta^{4}} \int_{-\infty}^{\infty} d s_{1} \int_{-\infty}^{\infty} d s_{2} \frac{\exp \left(-\left(\left|s_{1}\right|+\left|s_{2}\right|\right)-\frac{b_{0}^{2}}{2 \zeta^{2}}\left(s_{1}-s_{2}\right)^{2}\right)}{1+\frac{b_{0}^{4}}{4 \zeta^{2}}\left(\frac{s_{1}}{B+\frac{\Delta B}{2}}-\frac{s_{2}}{B-\frac{\Delta B}{2}}\right)^{2}} .
$$

The exponent $\exp \left[-\frac{b_{0}^{2}}{2 \zeta^{2}}\left(s_{1}-s_{2}\right)^{2}\right]$ follows from the first identity Eq. (11), while the denominator emerges from the second identity.

As a next step we perform the integration over the difference $s_{1}-s_{2}$. This integration can be performed explicitly using the fact that $\zeta \ll b_{0} \ll B$. Upon this integration, the average Eq. (12) can be presented in the form $\frac{\sqrt{2 \pi}}{4 \zeta^{3} b_{0}} \Phi_{\|}\left(\frac{\Delta B}{B_{c}}\right)$, where the dimensionless function $\Phi_{\|}$ is defined as

$$
\Phi_{\|}(z)=\int_{0}^{\infty} d x \frac{e^{-x}}{1+z^{2} x^{2}},
$$

and $B_{c}=4 B^{2} \zeta / b_{0}^{2}$. The argument of the function $\Phi$ imposes a characteristic "period" of mesoscopic fluctuations: $\delta B \sim \frac{B^{2} \zeta}{b_{0}^{2}}$.

Eq. (13) suggests that the period of the mesoscopic fluctuations grows quadratically with $B$. We tested this result by a numerical simulation. For this simulation we chose $N=10^{4}$ random values of $\boldsymbol{b}$ with $\mathrm{rms} b_{0}=$ $10^{2} \zeta^{-1}$. For each set of the local hyperfine fields the sum $\sum_{n=1}^{N} \delta I_{n}(\boldsymbol{B})$, where $\delta I_{n}$ is given by Eq. (6) was evaluated. The results of simulation are shown in Fig. 2. Mesoscopic fluctuations and growth of their period with $B$ are apparent.

Our consideration applies for $\delta B \ll B$. This condition suggests that for measuring the fluctuations one must work in the domain $b_{0} \ll B \ll b_{0}^{2} / \zeta$. The correlator Eq. 
(13) is plotted in Fig. 1, For small $\delta B \ll B_{c}$ it behaves as $1-\left(\frac{\delta B}{B_{c}}\right)^{2}$, and falls off slowly, as $\frac{\pi B_{c}}{2 \delta B}$, for $\delta B \gg B_{c}$.

For the perpendicular case, we can simplify $\Delta$ as $\Delta \approx\left(\boldsymbol{b}_{1}-\boldsymbol{b}_{2}\right) \cdot \boldsymbol{n}$. This is because the $\boldsymbol{B}$-dependence of $\Delta$ enters via the orientation, $\boldsymbol{n}$. Performing the same decoupling (Eq. (9)) as for the parallel case, instead of the double integral in Eq. (12) we get now

$$
\left\langle\frac{1}{\left(\Delta\left(\boldsymbol{B}+\frac{\Delta \boldsymbol{B}}{2}\right)^{2}+\zeta^{2}\right)\left(\Delta\left(\boldsymbol{B}-\frac{\Delta \boldsymbol{B}}{2}\right)^{2}+\zeta^{2}\right)}\right\rangle=\int \frac{d s_{1}}{2 \zeta^{2}} e^{-\left|s_{1}\right|} \int \frac{d s_{2}}{2 \zeta^{2}} e^{-\left|s_{2}\right|} \exp \left\{-\frac{b_{0}^{2}}{2 \zeta^{2}}\left(s_{1}^{2}+s_{2}^{2}-2 s_{1} s_{2} \cos \varphi\right)\right\} .
$$

We again see that by virtue of the relation $b_{0} \gg \zeta$, the difference $\left(s_{1}-s_{2}\right) \sim \frac{\zeta}{b_{0}}$ is small. This allows us to integrate over $s_{1}-s_{2}$ and reduce Eq. (14) to $\frac{\sqrt{2 \pi}}{4 \zeta^{3} b_{0}} \Phi_{\perp}\left(\frac{\varphi}{\varphi_{c}}\right)$, where $\varphi_{c}=\frac{2 \sqrt{2} \zeta}{b_{0}} \ll 1$ and the function $\Phi_{\perp}(z)$ is defined as

$$
\Phi_{\perp}(z)=\int_{0}^{\infty} d x \exp \left[-x-z^{2} x^{2}\right]
$$

The correlator is plotted in Fig. 1] At $\varphi \gg \varphi_{c}$, it falls off as $\varphi_{c} / \varphi$. In general, the correlator, Eq. (14) is a periodic function of $\varphi$; had we not used the small- $\varphi$ expansion it would go through a minimum at $\varphi=\pi / 2$ and "revive" at $\varphi=\pi$.

Our results related to mesoscopics can be summarized in the following expression

$$
\frac{\left\langle\delta I\left(\boldsymbol{B}-\frac{\Delta \boldsymbol{B}}{2}\right) \delta I\left(\boldsymbol{B}+\frac{\Delta \boldsymbol{B}}{2}\right)\right\rangle}{I(\infty)^{2}}=\frac{\sqrt{2 \pi} \zeta}{4 N b_{0}}\left\{\begin{array}{l}
\Phi_{\|}\left(\frac{\Delta B}{B_{c}}\right), \Delta \boldsymbol{B} \| \boldsymbol{B} \\
\Phi_{\perp}\left(\frac{\varphi}{\varphi_{c}}\right),{ }_{\Delta} \boldsymbol{B} \perp \boldsymbol{B}
\end{array}\right.
$$

\section{DISCUSSION}

- By choosing a simple transport model for an organic semiconductor device, and adopting the assumption 13,18 that recombination proceeds exclusively from the singlet state, we were able to demonstrate mesoscopic fluctuations in the OMAR response in the domain $B \gg b_{0}$, where the average current is saturated, and predict their characteristic magnitude and period. Our theory is based on an observation that in this $B$-domain there exists a strong separation between slow and fast components of the PP spin-dynamics. As a result of this separation, the $S-T_{0}$ beating becomes decoupled, which, in turn, leads to a dramatic recombination slow down which originates from PP "trapping" in the $T_{0}$ state. Since this underlying physics is so general, any transport model in a small device with few junctions should exhibit mesoscopic fluctuations. What is really required for mesoscopic features to emerge in $I(B)$ is that the transport is in the regime of "slow-hopping", namely $b_{0} \gg \zeta$.
It is in this regime when sparse PPs, for which the $\boldsymbol{b}_{h}$ projections on the external field almost coincide, play a distinguished role.

Mesoscopic effect persists when recombination from triplet is also allowed. Important is that the recombination times from singlet and triplet PPs differ.

- In the consideration we assumed that the time, $\tau_{D}$ of the pair formation is equal to the time of pair disassembly. This requirement is not restrictive for mesoscopics. What is important for mesosopics is that both times exceed the recombination time, $\tau$. In fact, this requirement is a general requirement for spin-dependent recombination, which is at the core of the OMAR effect.

- As we mentioned above the transport model adopted in the present paper is quite similar to bipolaron model of transport put forward in Ref. 13. Replacement of bipolaron formation by recombination does not bring in any new qualitative features. Thus the mesoscopic fluctuations demonstrated in the present paper can be viewed as a correction due to the local environment to the average current emerging from the mechanism Ref. 13 .

- Regarding experimental verification of the predicted mesoscopic fluctuations, we note that there might be an alternative (to decreasing the size) way to bring samples into a mesoscopic regime. It was demonstrated in Ref. 28 that tin-doped indium oxide (ITO) electrodes exhibit sharp pillars with areal density of $\sim 1 \mu \mathrm{m}^{-2}$. These pillars may cause additional inhomogeneity of the local conductivity and even define high-conductivity channels in the active layer. If this is the case, one can estimate from the data in Ref. 28, that a small OLED with area of $\sim 10^{-2} \mathrm{~cm}^{2}$ will show mesoscopic fluctuations of $\frac{\delta I}{I} \sim 10^{-3}$.

\section{ACKNOWLEDGEMENTS}

We are grateful to E. Erenfreund for useful discussions. This work was supported by NSF through MRSEC DMR-1121252 and DMR-1104495. 
${ }^{1}$ K. M. Salikhov, Y. N. Molin, R. Z. Sagdeev, and A. L. Buchachenko, in Spin Polarization and Magnetic Effects in Radical Reactions, edited by Y. N. Molin (Elsevier, Amsterdam, 1984), pp. 32116, and the review U. E. Steiner and T. Ulrich, Chem. Rev. 89, 51 (1989).

${ }^{2}$ K. Schulten and P. G. Wolynes, J. Chem. Phys. 68, 3292 (1978).

3 T. L. Francis, Ö. Mermer, G. Veeraraghavan, and M. Wohlgenannt, New J. Phys. 6, 185 (2004).

4 Ö. Mermer, G. Veeraraghavan, T. L. Francis, Y. Sheng, D. T. Nguyen, M. Wohlgenannt, A. Khler, M. K. Al-Suti, and M. S. Khan, Phys. Rev. B 72, 205202 (2005).

${ }^{5}$ Y. Sheng, T. D. Nguyen, G. Veeraraghavan, O. Mermer, M. Wohlgenannt, S. Qiu, and U. Scherf, Phys. Rev. B 74, 045213 (2006).

6 V. N. Prigodin, J. D. Bergeson, D. M. Lincoln, and A. J. Epstein, Synth. Met. 156, 757 (2006).

7 P. Desai, P. Shakya, T. Kreouzis, and W. P. Gillin, Phys. Rev. B 76, 235202 (2007).

8 F. J. Wang, H. Bässler, and Z. Valy Vardeny, Phys. Rev. Lett. 101, 236805 (2008).

9 T. D. Nguyen, G. Hukic-Markosian, F. Wang, L. Wojcik, X.-G. Li, E. Ehrenfreund, and Z. V. Vardeny, Nat. Mater. 9, 345 (2010).

10 T. D. Nguyen, B. R. Gautam, E. Ehrenfreund, and Z. V. Vardeny, Phys. Rev. Lett. 105, 166804 (2010).

11 Tho D. Nguyen, T. P. Basel, Y.-J. Pu, X-G. Li, E. Ehrenfreund, and Z. V. Vardeny, Phys. Rev. B 85, 245437 (2012).

12 F. L. Bloom, W. Wagemans, M. Kemerink, and B. Koopmans, Phys. Rev. Lett. 99, 257201 (2007).

13 P. A. Bobbert, T. D. Nguyen, F. W. A. van Oost, B. Koopmans, and M. Wohlgenannt, Phys. Rev. Lett. 99, 216801 (2007).

14 W. Wagemans, F. L. Bloom, P. A. Bobbert, M. Wohlgenannt, and B. Koopmans, J. Appl. Phys. 103, 07F303
(2008).

15 F. L. Bloom, M. Kemerink, W. Wagemans, and B. Koopmans, Phys. Rev. Lett. 103, 066601 (2009).

16 S. P. Kersten, A. J. Schellekens, B. Koopmans, and P. A. Bobbert, Phys. Rev. Lett. 106, 197402 (2011).

17 W. Wagemans, A. J. Schellekens, M. Kemper, F. L. Bloom, P. A. Bobbert, and B. Koopmans Phys. Rev. Lett. 106, 196802 (2011).

18 N. J. Harmon and M. E. Flatté, Phys. Rev. Lett. 108, 186602 (2012); Phys. Rev. B 85, 075204 (2012); Rev. B 85, 245213 (2012).

19 L. I. Glazman and K. A. Matveev, Zh. Eksp. Teor. Fiz. 94, 332 (1988) [Sov. Phys. JETP 67, 1276 (1988)].

20 M. E. Raikh and I. M. Ruzin, in Mesoscopic Phenomena in Solids, edited by B. L. Altshuller, P. A. Lee, and R. A. Webb (Elsevier, New York, 1991), p. 315.

21 R. J. F. Hughes, A. K. Savchenko, J. E. F. Frost, E. H. Linfield, J. T. Nicholls, M. Pepper, E. Kogan, and M. Kaveh, Phys. Rev. B 54, 2091 (1996).

22 D. R. McCamey, S.-Y. Lee, S.-Y. Paik, J. M. Lupton, and C. Boehme Phys. Rev. B 82, 125206 (2010).

23 A. V. Khaetskii, D. Loss, and L. I. Glazman, Phys. Rev. Lett. 88, 186802 (2002).

24 T. V. Shahbazyan and M. E. Raikh, Phys. Rev. B 49, 17123 (1994).

25 J. König, Y. Gefen, and G. Schön, Phys. Rev. Lett. 81, 4468 (1998).

26 M. G. Schultz and F. von Oppen, Phys. Rev. B 80, 033302 (2009).

27 R. C. Roundy and M. E. Raikh, Phys. Rev. B 87, 195206 (2013).

28 G. Liu, J. B. Kerr, and S. Johnson, Synth. Met. 144, 1 (2004). 